\title{
The uptake and use of a minimum data set (MDS) for older people living and dying in care homes: a realist review
}

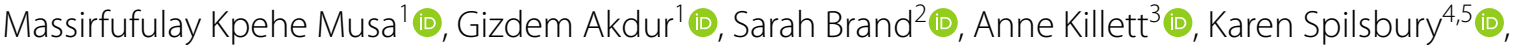

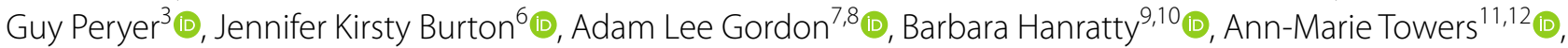 \\ Lisa Irvine ${ }^{1}$, Sarah Kelly ${ }^{13} \mathbb{0}$, Liz Jones ${ }^{14}$, Julienne Meyer ${ }^{15}$ and Claire Goodman ${ }^{1,16^{*}}$ (D)
}

\begin{abstract}
Background: Care homes provide long term care for older people. Countries with standardised approaches to residents' assessment, care planning and review (known as minimum data sets (MDS)) use the aggregate data to guide resource allocation, monitor quality, and for research. Less is known about how an MDS affects how staff assess, provide and review residents' everyday care. The review aimed to develop a theory-driven understanding of how care home staff can effectively implement and use MDS to plan and deliver care for residents.

Methods: The realist review was organised according to RAMESES (Realist And Meta-narrative Evidence Synthesis: and Evolving Standards) guidelines. There were three overlapping stages: 1) defining the scope of the review and theory development on the use of minimum data set 2) testing and refining candidate programme theories through iterative literature searches and stakeholders' consultations as well as discussion among the research team; and 3) data synthesis from stages 1 and 2. The following databases were used MEDLINE via OVID, Embase, CINAHL (Cumulative Index to Nursing and Allied Health Literature), ASSIA [Applied Social Sciences Citation Index and Abstracts]) and sources of grey literature.

Results: Fifty-one papers informed the development of three key interlinked theoretical propositions: motivation (mandates and incentives for Minimum Data Set completion); frontline staff monitoring (when Minimum Data Set completion is built into the working practices of the care home); and embedded recording systems (Minimum Data Set recording system is integral to collecting residents' data). By valuing the contributions of staff and building on existing ways of working, the uptake and use of an MDS could enable all staff to learn with and from each other about what is important for residents' care

Conclusions: Minimum Data Sets provides commissioners service providers and researchers with standardised information useful for commissioning planning and analysis. For it to be equally useful for care home staff it requires key activities that address the staff experiences of care, their work with others and the use of digital technology.
\end{abstract}

Registration: PROSPERO registration number CRD42020171323.

Key words: Older people care, long-term care, care home, standardised care, minimum-data-set

*Correspondence: c.goodman@herts.ac.uk

${ }^{1}$ Centre for Research in Public health and Community Care (CRIPACC),

School of Health and Social Work, University of Hertfordshire, Hatfield, UK

Full list of author information is available at the end of the article

\section{Background}

In the UK, an estimated 1.6 million people are aged 85 years and above [1]. Longevity of the oldest old (age 85 years and above), is associated with higher levels of 
dependency and projected need for long term care [2]. About 420,000 older people in England and Wales live in care homes [3, 4]. Care home is a generic term referring to facilities in which people live together with staff onsite 24 hours a day to provide care, with some homes having on-site registered nurses [5]. Whilst care homes may provide short-term respite care, for most people they are the sole place of residence or home. The care home population encompasses some of the most vulnerable members of society, with approximately $70 \%$ living with cognitive impairment $[6,7]$. The health and care needs of this population are met by a range of health and social care staff working in, or with, care homes. Information about residents' characteristics, needs and services they receive sits in multiple unaligned health and social care databases. Without a national core dataset based on resident-level information very little, outside of research evidence is known about this population [8]. An increasing post pandemic priority is ensuring efficient and effective sharing of resident data for the purposes of care, planning and evaluating services.

The COVID-19 pandemic in the UK highlighted the consequences of having no nationally mandated data collection on care home residents or links with National Health Service (NHS) records [8-10]. Not having standardised and accessible information about care home residents' medical history, service use and care needs, had a negative impact on the public health response for this population [11]. This delayed recognition of excess mortality in care homes and policy measures that were care home specific for infection prevention strategies for residents and staff [12]. All care homes collect substantial amounts of data about their residents and many use validated assessments, for example for nutritional status, falls risk and dependency levels [13].The need for a common approach to data capture or links with health care data sets is a policy priority and an implementation challenge [14],

There are different versions of MDS used in long term care around the world (for example the MDS 3.0 ( Saliba and Buchanan )[15] used in USA and International Resident Assessment Instrument (Inter-RAI) used extensively in Canada and adapted for different care systems in New Zealand, parts of Australia and some countries of mainland Europe [16], . The use of an MDS is often but not always mandated and/or linked to national reimbursement. For example, in the USA Medicare reimbursement is based on responses from the MDS 3.0 to determine residents' care needs. MDS support comprehensive assessment of care home residents and linked care planning, enable multidisciplinary working, quality assessment, and inform commissioning of services [1724]. There are administrative costs and concerns about the burden that they place on staff, the depersonalisation of care and if consistency across care systems can be achieved $[25,26]$.

Previous research in the UK has tested existing standardised approaches to resident assessment and data [27, 28]. but there is limited work on what needs to be in place for effective implementation. The starting assumption of this realist review is that decision-making for care home residents' care can be enhanced through the application of data that can be used by a range of stakeholders [29]. The review's particular focus is on how long-term care settings make the transition to standardised approaches to data collection, and how its use impacts on staff work, time away from care, knowledge of the care home residents, working with other healthcare professionals.

An MDS is defined as a standardised account of the demographic, social, and health characteristics and needs of older people living in long-term care (care home) settings.

\section{Aim}

To develop a theory-driven understanding of how care home staff can effectively implement and use MDS to plan and deliver care for residents.

\section{Objectives}

1. Develop a programme theory describing contexts that support the uptake and use of an MDS in care homes.

2. Identify in what circumstances the use of an MDS produces improved outcomes (including resource use) for an individual resident, their family, and the care home staff and their employing organisation.

\section{Methods}

A realist review to develop a theory of what needs to be in place for effective MDS uptake and use at the resident level of care [30-33]. This theory-driven approach to reviewing research evidence on complex social interventions can provide an explanatory analysis of how and why interventions work (or not) in particular contexts or settings, as well as unintended consequences [32, 34].

Realism asserts that it is not interventions that create change; rather, it is the people involved and their responses [32, 35, 36]. This review uses the evidence to identify and test the interactions between contexts, mechanisms and outcomes (or 'CMOs'), to provide an explanatory account of how an intervention works (Table 1).

This review draws on practical 'how-to' guidance [34, 37], and follows publication standards (Realist And 
Table 1 Glossary of realist terms in this review

Contexts (C) - Are often 'the 'backdrop' of programmes and research... broadly understood as any condition that triggers and/or modifies the behaviour of a mechanism [39].

Mechanisms (M) - are not observed directly but account for what it is about programmes that makes them work, characterised as "underlying entities, processes or structures which operate in particular contexts to generate outcomes of interest" ([40], p.368). Mechanisms are the responses of those involved in an intervention/programme to the resources or opportunities offered by that intervention/programme. Responses may include thoughts, feelings or actions. They are activated or inhibited by circumstances or contexts that then have an effect ([31], p.xvii).

Outcomes (0) - are strategies of the intervention/programme (planned or unplanned, visible or not); result of the interaction between a mechanism and its triggering context [31,41].

Programme theories - an overarching theory or model of how a programme, or an intervention is expected to work, and it helps to explain (some of) 'how and why, in the "real world", a specific programme "works", for whom, to what extent and in which contexts' [37, 40].

Demi-regularities - a"prominent recurrent patterns of contexts and outcomes... in the data" ([33], p. 9).

Context-Mechanism-Outcome (CMO) - CMO an heuristic used to explain generative causation, which help to explain the relationship between a context, mechanism, and an outcome of interest in a particular programme [42]. It demonstrates the causal components that explain what works in an intervention/programme for who, why and in which circumstances [31].

Meta-narrative Evidence Syntheses: Evolving Standards (RAMESES)) guidance [32]. A more detailed account of the methods is published elsewhere [38].

Realist reviews go beyond identification of barriers and facilitators to provide a theory-driven explanation of what needs to be in place for implementation [30-32]. The review is organised in three stages (Fig. 1). First, a scoping of the literature to identify care home specific work on the acceptance and use of MDS in care home settings. Next, a theory driven review of the evidence, plus interviews with key stakeholders to test and refine theories that explain the use of an MDS and linked resident and staff outcomes. Finally, a synthesis of the evidence to establish how and when the use of an MDS achieves different outcomes for residents,

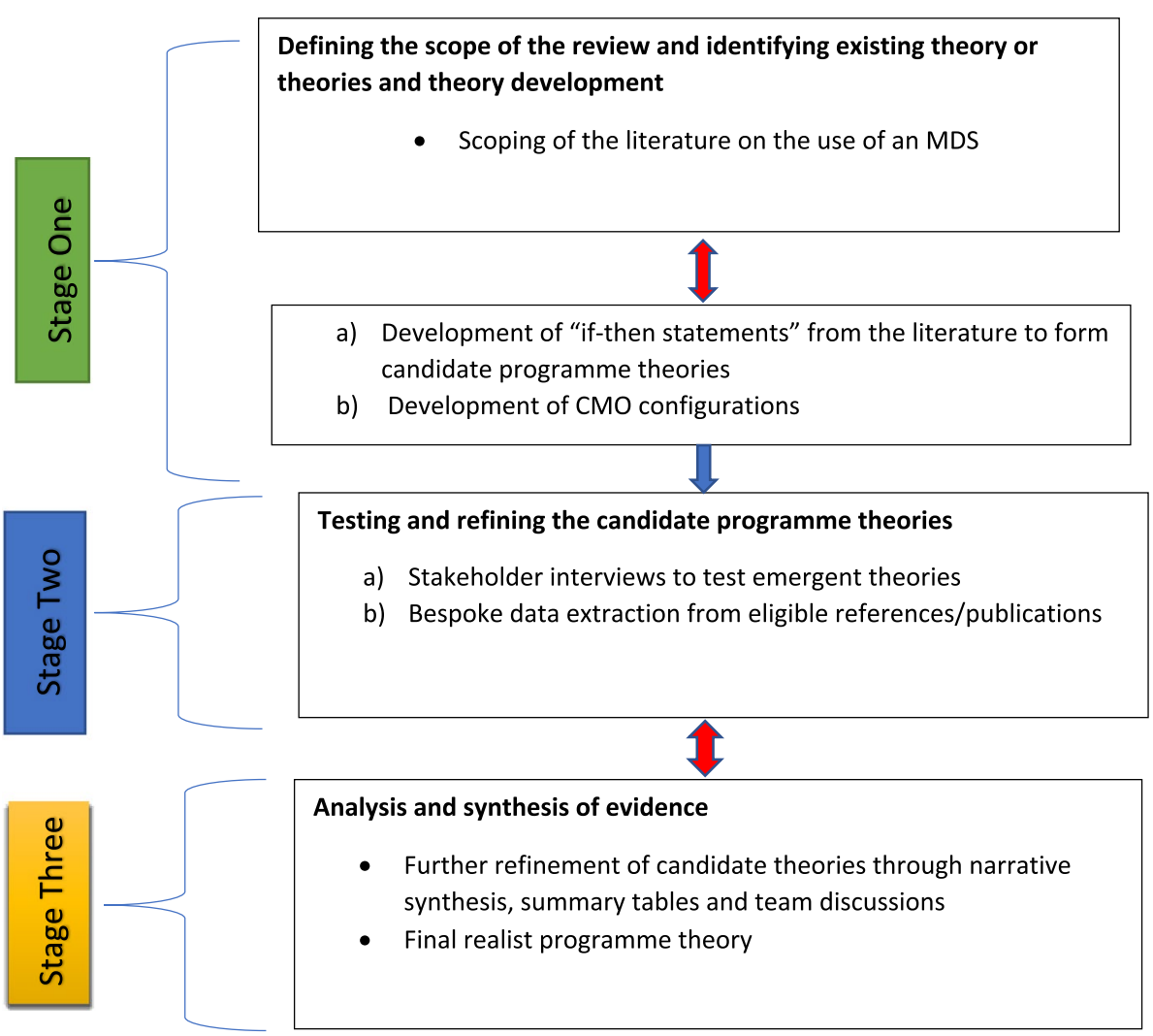

Fig. 1 The three-staged approach to the synthesis 
families, staff, and organisations and presentation of a final programme theory.

\section{Changes from the submitted protocol in the review process}

Realist review is an iterative process; thus, adjustments were made to the review protocol [38] in the light of emerging or new lines of enquiry. The intention had been at the beginning of the review to conduct eight interviews with key stakeholders with experience of implementing and using MDS for care work. There were changes to the focus and timing of interviews to explore in more detail how staff engaged with new ways of capturing data and allow a greater emphasis on whether findings resonated with those of the interviewees. Stakeholders' availability and recruitment was affected by the Covid-19 pandemic [43]. Four theory-driven interviews were completed (care home manager, care home staff member, international researcher with experience of implementing MDS and care home board member) (Online supplementary 1). Consequently, more time was given to drawing on the experiences and knowledge of the wider research team and study steering group of what supports data collection and staffs' use of standardised measures in care homes. The study steering group membership included three leaders of care home organisations and their representative bodies, two resident representatives, three care home researchers, a clinician working with care homes and three long term care data specialists.

Ethical approval for interviews was received from the University of Hertfordshire Ethics committee (HSK/SF/ $\mathrm{UH} / 04169$ ).

All methods were performed in accordance with the relevant guidelines and regulations.

\section{Stage 1: Defining the scope of the review, identifying existing theories (concept mining and candidate theory (theories) development)}

The exploratory scoping of the literature began with evidence nested within a larger review on assessment and outcome measure used in care home research (PROSPERO reference: CRD42020155923). Between January to July 2020, we searched bibliographic databases MEDLINE via OVID, Embase, CINAHL (Cumulative Index to Nursing and Allied Health Literature), ASSIA [Applied Social Sciences Citation Index and Abstracts]) and sources of grey literature. We included literature published in English language over the last ten years for and applied terms such as care homes, skilled nursing facilities, long-term care facilities, and nursing homes, and then combined those terms with others such as MDS,
inter-RAI, Geriatric Assessment, and Research Assessment Instrument (Online supplementary 2).

The review drew on evidence from a wide range of sources [30,35]. A paper was included if the evidence was sufficiently detailed to be assessed "good enough and relevant" implementation and use of MDS in care homes $[38,44]$.

The data extraction and quality appraisal of included documents were done simultaneously [45]. A series of 'if-then' statements based on the evidence (Online supplementary 3), mapped possible causal relationships that were discussed across the research team and refined as CMO configurations. These guided the interviews with stakeholders and the theory testing review work in stage two.

\section{Stage 2: Candidate theories testing and refinement through further iterative searches}

Based on the theoretical propositions derived from the scoping work, search terms were reviewed. The database searches in Stage 1 (described above) were extended with lateral searches and forward citations of relevant documents, to capture studies on digital engagement, care home cultures that support uptake of MDS and additional implementation studies (Fig. 2). A bespoke structured data extraction form, based on the CMOs, captured how the MDS/assessment tool was used at the resident level of care.

Interviews with stakeholders and discussions across the research team discussed how the theories resonated with them as experts and possible alternative explanations relevant for the successful development and use of an MDS.

\section{Stage 3: Data analysis and synthesis processes}

Data analysis focused on how the evidence built upon, refuted or provided alternative explanations for the CMOs. First, observable patterns in context and outcome (demi-regularities) detectable within and among the data were reviewed and formed into a list of CMOs (Table 1). From this, three consolidated programme theories (PTs) were formed (Fig. 3) to explain how the use and uptake of MDS worked, for whom and in what circumstances. The way the CMOs were organised to capture how MDS may work at the organisational, staff and resident levels of care is presented in Fig. 4.

\section{Results}

The findings are presented in linear way, the analyses however, involved iterative processes as shown in various figures containing double arrows. 
Stage-1

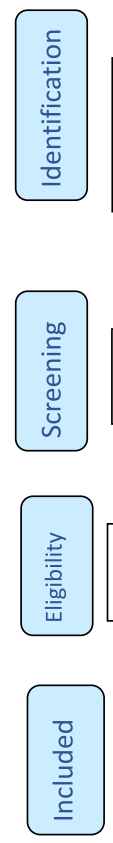

Records identified through database searching ( $n=479$ )

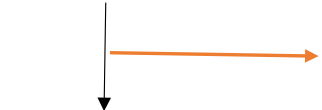

447 Titles and abstracts screened

194 Full-text article assessed for eligibility

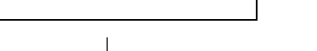

32 Records removed as duplicates レニニニニニニニニニニニニニニニー

253 Records excluded for being irrelevant

-

159 articles excluded for not providing information on research question, inaccessible, written in non-English language, or reoccurring themes

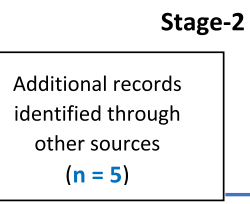

Additional records identified through other sources

$$
(n=5)
$$

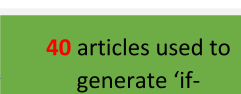
then'statements CMOs

Stage-2

Stakeholders and experts' consultation

\section{(semi-structured}

interviews)

Fig. 2 Document flow and review processes: conceptual diagram of database searches, snowballing searches, and iterative cycles

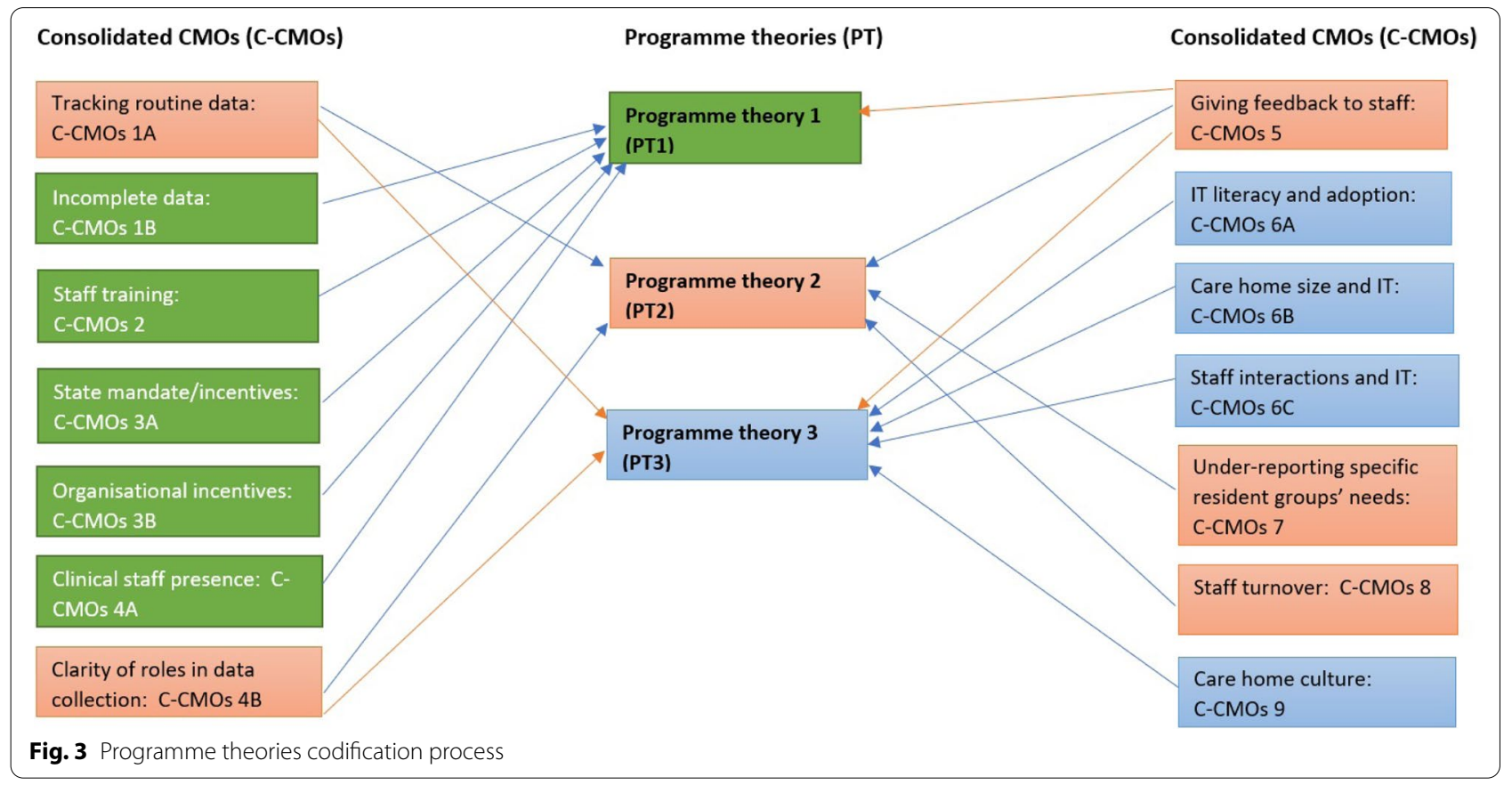




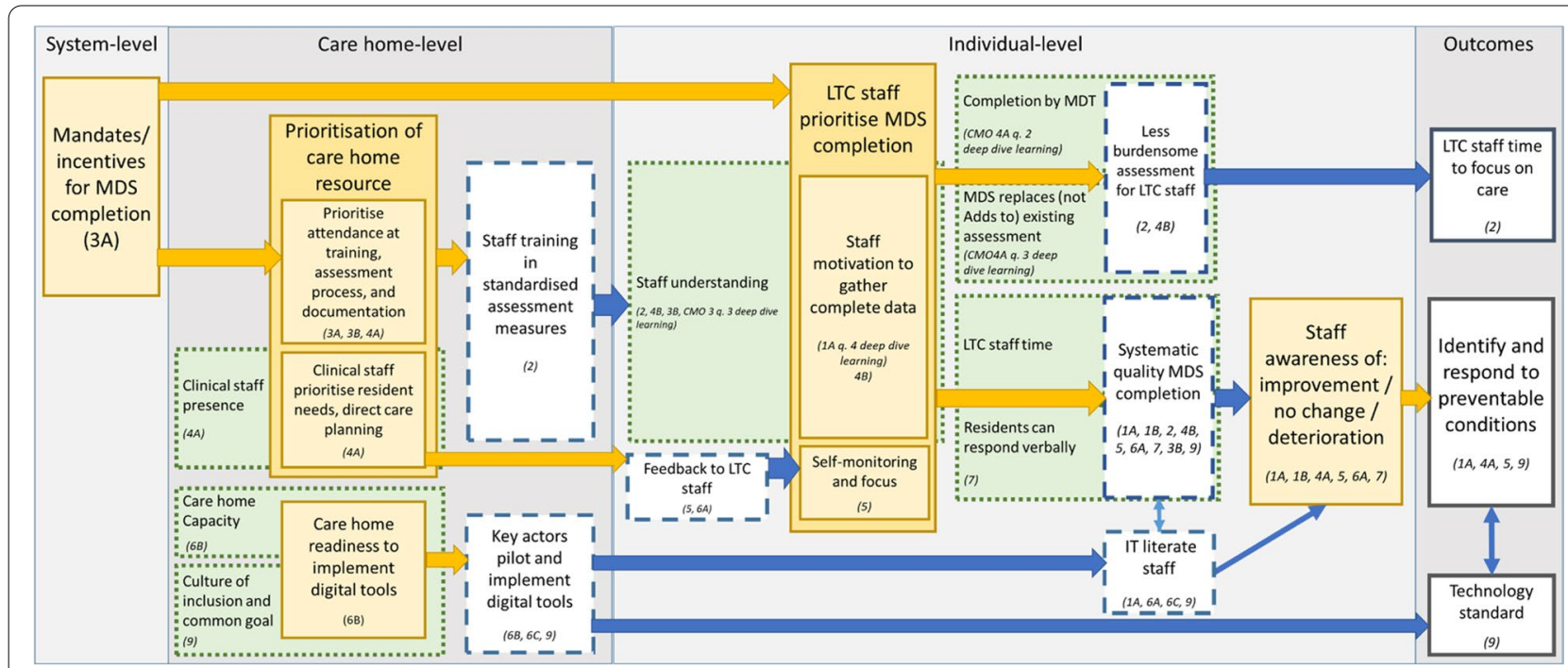

Fig. 4 Mapping of CMOs and outcomes

\section{Characteristics of included studies}

Of the 479 records initially retrieved from electronic database search, 194 were included for full-text screening (Fig. 2). Most papers that completed secondary data analysis of MDS were excluded because they did not provide any data on the use of MDS within the care home. Studies were included that had a population focus but also discussed the quality of reporting, and anomalies, for example for residents from different ethnic backgrounds [46-48] or in receipt of different types of funding [18]. Thirty-five papers were included from the search. Five additional publications were identified through lateral searches; 40 full papers were included in the scoping review (Fig. 2). Of these, over two thirds came from North America (USA $(n=25)$, Canada $(n=7)$ with one or two papers from each of Australia $(n=2)$, Taiwan $(n=2)$, Italy $(n=1)$, New Zealand $(n=1)$, Norway $(n=1)$, and the UK $(n=1)$ (Online supplementary 4).

Thirty-three 'if-then' statements were formed from literature data (Online supplementary 3). Of these 'if- then' statements, 14 recurring themes in the data formed the basis of our consolidated CMO (C-CMO) configurations (Table 2). We then carried out further background literature, lateral and forward citations and theory driven searches for phase two and identified additional 16 references, 11 of which were papers on implementation research in care homes (Fig. 2). We finally codified the nine $\mathrm{C}$-CMOs into three interlinking programme theories (Fig. 3).

\section{Stakeholder interviews}

Each interview lasted 45 mins - 1 hour. Key issues identified from the interviews that informed the interpretation of the scoping review findings and final analysis were: feedback to staff on residents' status; frequency of data capture in a working day; staff members' pre-existing knowledge of the resident; consistency of care among staff and how this influences their ability use digital approaches to data capture. A recurring topic was how to resolve the need to complete MDS aggregated at the care home level, for example, for planning and audit and MDS use for individual residents' daily care.

\section{Programme theory}

Three key inter-linked theoretical propositions, based on nine consolidated CMOs (C-CMOs), articulate what supports the uptake and use of MDS by frontline staff (Fig. 3). These focused on how mandates and incentives, the involvement and oversight of clinicians in the use of MDS and staff skills and readiness to engage with digital technology led to meaningful data capture (or not) (Table 3).

The three key theoretical propositions (Box 2) are inter-linked. The programme theory showing how these relate to each other and to the desired outcome of MDs are shown in Fig. 4 (see online supplementary file 5 for a more detailed mapping of the contexts, mechanisms and outcomes involved in moving from mandates to outcomes). The main mechanism to achieve the identification of, and responsiveness to, preventable conditions in care home residents $(\mathrm{O})$ was frontline staff awareness of residents improvement or deterioration 
Table 2 List of CMOs

\begin{tabular}{|c|c|c|c|}
\hline \multirow[t]{2}{*}{$\begin{array}{l}\text { Tracking with routine } \\
\text { data } \\
\text { (1A) }\end{array}$} & \multirow{2}{*}{$\begin{array}{l}\text { Systematic identification of residents' } \\
\text { functional needs with routine data } \\
\text { increases staff awareness, accuracy and } \\
\text { supports consistent staff responses, which } \\
\text { as a result makes tracking resident } \\
\text { improvement (or not) over time easier, and } \\
\text { avoids preventable conditions e.g. } \\
\text { pressure sores, pain, falls unnecessary } \\
\text { referrals and admissions }\end{array}$} & $\begin{array}{l}\text { State mandates I } \\
\text { incentives } \\
(3 \mathrm{~A})\end{array}$ & $\begin{array}{l}\text { The mandates and/or incentives for the } \\
\text { completion of MDS means that the } \\
\text { assessment process and attendance at } \\
\text { trainings are prioritised, therefore there are } \\
\text { more accurate summaries of resident } \\
\text { status. People in the wider system using } \\
\text { similar systems }\end{array}$ \\
\hline & & \multirow{2}{*}{$\begin{array}{l}\text { Organisational } \\
\text { incentives } \\
\text { (3B) }\end{array}$} & \multirow{2}{*}{$\begin{array}{l}\text { When the staff members are expected to } \\
\text { make further contributions every time a } \\
\text { resident record is accessed, the } \\
\text { documentation is prioritised, and staff } \\
\text { understand better how their data is used. } \\
\text { As a result, data quality and clarity on } \\
\text { documentation improve. }\end{array}$} \\
\hline \multirow[t]{2}{*}{$\begin{array}{l}\text { Incomplete data } \\
\text { (1B) }\end{array}$} & \multirow{2}{*}{$\begin{array}{l}\text { Incomplete MDS means that staff does not } \\
\text { recognise some components, and } \\
\text { therefore some symptoms are not picked } \\
\text { up }\end{array}$} & & \\
\hline & & \multirow{2}{*}{$\begin{array}{l}\text { Clinical staff presence } \\
(4 \mathrm{~A})\end{array}$} & \multirow[b]{2}{*}{$\begin{array}{l}\text { When clinically qualified staff is involved in } \\
\text { resident assessments, they prioritise } \\
\text { needs and direct care planning and review } \\
\text { progress with non-clinical staff. This } \\
\text { results in the specific needs of the } \\
\text { residents (e.g. depression, incontinence) } \\
\text { being identified and acted upon }\end{array}$} \\
\hline \multirow[t]{2}{*}{$\begin{array}{l}\text { Staff training } \\
(2)\end{array}$} & \multirow{2}{*}{$\begin{array}{l}\text { Staff who are trained in the completion of } \\
\text { standardised assessment measures are } \\
\text { more aware of improvement and } \\
\text { deterioration and understand the link } \\
\text { between assessment and outcomes, } \\
\text { therefore they have more time to focus on } \\
\text { care, assess the residents in a less } \\
\text { burdensome way and the assessment } \\
\text { quality improves }\end{array}$} & & \\
\hline & & $\begin{array}{l}\text { Clarity of roles in data } \\
\text { collection } \\
\text { (4B) }\end{array}$ & $\begin{array}{l}\text { lack of clarity regarding roles and } \\
\text { responsibilities for data collection } \\
\text { demotivates staff and it causes data } \\
\text { duplication and lack of quality }\end{array}$ \\
\hline $\begin{array}{l}\text { Giving feedback to } \\
\text { staff } \\
\text { (5) }\end{array}$ & $\begin{array}{l}\text { Feedback given to staff encourages self- } \\
\text { monitoring and focus, and this translates } \\
\text { into reduction in residents' avoidable } \\
\text { symptoms e.g. pain, depression, falls. }\end{array}$ & \multirow[t]{2}{*}{$\begin{array}{l}\text { Under reporting } \\
\text { specific resident } \\
\text { groups' needs } \\
\text { (7) }\end{array}$} & \multirow{2}{*}{$\begin{array}{l}\text { The symptoms, conditions and needs by } \\
\text { residents who cannot respond verbally } \\
\text { because of cognition, language or cultural } \\
\text { background are not captured as effectively } \\
\text { and this leads to staff underreporting and } \\
\text { missing needs. This results in misreporting } \\
\text { of resident need and misclassification of } \\
\text { symptoms, such as pain. }\end{array}$} \\
\hline \multirow[t]{3}{*}{$\begin{array}{l}\text { IT literacy and } \\
\text { IT adoption } \\
\text { (6A) }\end{array}$} & \multirow{3}{*}{$\begin{array}{l}\text { When IT literate staff use digital } \\
\text { assessment tools in real time with } \\
\text { resident, they engage better with the } \\
\text { information and are more responsive to } \\
\text { feedback. This results in the identification } \\
\text { of missed care, improvement in the quality } \\
\text { of care, trend identification in common } \\
\text { conditions and the accurate capturing of } \\
\text { residents' characteristics. }\end{array}$} & & \\
\hline & & \begin{tabular}{|l} 
Staff turnover \\
$(8)$
\end{tabular} & $\begin{array}{l}\text { The low staff turnover might increase the } \\
\text { staff familiarity with residents. This makes } \\
\text { MDS data more meaningful and MDS } \\
\text { becomes more likely to pick up pain. }\end{array}$ \\
\hline & & \multirow{3}{*}{$\begin{array}{l}\text { Care home culture } \\
\text { (9) }\end{array}$} & \multirow{3}{*}{$\begin{array}{l}\text { When the care home has a culture of } \\
\text { inclusion and a common understanding of } \\
\text { what is to be achieved, the key actors are } \\
\text { involved and create dialogue throughout a } \\
\text { technology implementation, and the } \\
\text { technologies and clinical practices improve } \\
\text { iteratively. It also leads to the pilot-testing } \\
\text { of technologies, and the planning of } \\
\text { coaching activities. As a result, the } \\
\text { successful implementation of digital } \\
\text { monitoring technologies is achieved, and } \\
\text { there is less resistance towards the new } \\
\text { technologies. }\end{array}$} \\
\hline $\begin{array}{l}\text { Care home size } \\
\text { and IT } \\
(6 \mathrm{~B})\end{array}$ & $\begin{array}{l}\text { The capacity of a care home can play a } \\
\text { role in how readily available they are in } \\
\text { implementing digital tools; the capacity of } \\
100-159 \text { beds in a care home results in } \\
\text { being less likely than other facilities to } \\
\text { implement an EHR (electronic health } \\
\text { record). }\end{array}$ & & \\
\hline $\begin{array}{l}\text { Staff interactions } \\
\text { and IT } \\
(6 \mathrm{C})\end{array}$ & $\begin{array}{l}\text { The unique interactions between staff } \\
\text { members may decrease with increased IT } \\
\text { adoption, and therefore residents } \\
\text { experience a decline in daily living } \\
\text { activities increase due to this loss. }\end{array}$ & & \\
\hline
\end{tabular}

Table 3 Three interlinked programme theories

Motivation: Mandates and incentives for MDS completion combined with MDS training and clinician involvement for care home staff motivates staff to complete MDS for residents and use this as the basis for discussion and care planning to identify residents' needs and review care.

Frontline staff monitoring: Completion of the MDS is built into the working practices, monitoring, and record systems of all staff (including visiting clinicians) involved in residents' care, with junior staff contributing to data entry. This creates an accessible comprehensive account of residents' needs, supports continuity of care, especially in instances of residents who cannot respond verbally because of cognition, language, or cultural background.

Embedded recording systems: When staff MDS recording systems are embedded as part of the care home approach to collecting resident data and staff are skilled in using digitally based systems to record residents' needs as part of a person-centred care process the accuracy and relevance of data will reflect residents' experiences and be used as the basis for care planning and review and reduce time away from providing care 
(M) (Fig. 4: C-CMOs 1A, 1B, 4A, 5, 6A, 7). This awareness $(M)$ depended upon frontline staff prioritising MDS completion, which depended upon the prioritisation of care home resource to achieve MDS completion which in turn, depended upon a system-level mandate for MDS completion. There are three key pathways from mandate to MDS completion: care home staff motivation (key theoretical proposition 1), care home staff self-monitoring and focus (key theoretical proposition 2), and IT literate care home staff using digital assessment tools in real time with residents (key theoretical proposition 3) (Box 2).

It was unclear how care home staff prior knowledge of residents' affected completion of an MDS. Low staff turnover for example, could either mean familiarity with residents, improved documentation of residents' needs or that important changes or significant pieces of information were missed.

The three key theoretical propositions are described in detail below.

\section{Motivation}

It is the motivation of frontline staff to gather complete data about residents that supports systematic and complete identification of residents' functional needs and ensures staff awareness of residents' changing status. This is reliant on staff understanding the link between their assessment and resident outcomes, their roles and responsibilities for data collection, and how their collected data will be used (Fig. 4).

Theory development suggested that frontline staff motivation can be increased through training in the completion of MDS and purpose of MDS data (Table 1). Most of the evidence reviewed was from North America. Completion of MDS in these settings is mandated by the federal government or the state. It is not discretionary. Whilst there was a sense in which staff might feel 'forced' to complete an MDS due to care home prioritisation of training in response to system-level mandates, this 'forcing' [22] could lead to the sustained use of MDS. It enabled staff to discover the benefits and thereby develop critical individual-level motivation to use the MDS in everyday practice. Here, the motivation might come initially from external mandates, but is then internalised and sustained through the process of engaging with MDS. Motivation was adversely affected where there was underinvestment in training or training that did not involve staff who were providing direct care. If the MDS presented as an administrative task this took the focus away from the resident. There were examples of how this led to incomplete collection of data, or data completion with groups of residents' needs not fully reported [49] [19, 25, 50]; (also, see Table 1).
Staff training by care homes depended upon prioritisation of team resource toward MDS completion. This included prioritising attendance at training, the assessment process (Fig. 4: C-CMO 3A), documentation (with staff being expected to edit resident records on each assessment; 3B), involvement in direct care planning, and review of frontline staff progress with clinical staff (which requires clinical staff in the care home at least some of the time; $4 \mathrm{~A}$ ).

Initial motivation to complete an MDS may rely on this training and care home level reinforcement of the utility of the data for improving resident care. In the long term, sustained motivation can develop through the feedback loop of staff witnessing changes in resident care due to systematic data collection and frontline staff having a shared confidence that their observations are valued. This may represent a critical aspect of the sustainability of MDS use in care homes. Completion of specific aspects of an MDS, e.g. continence and oral health care [51] illustrated how training affected staff engagement with an MDS. One review on MDS completion identified the needed interplay between competency in completing the MDS, training to support understanding of the categories of assessment, and review and staff engagement with managers and clinicians on residents' behalf over time. On page 21, the authors note:

"Data quality in the MDS will continue to reflect characteristics both of the instrument itself and of the assessors, their training and support. ...Consequently, ongoing education of clinical staff and health managers with respect to assessment practices and applications of the MDS is important" [52]. A plethora of evidence suggest the need for a range of training and support (Table 1), but it is unclear how some approaches provoked more lasting engagement than others. It may be fruitful for the sector to develop and test tools, such as frontline staff sharing groups, to further enable this feedback loop.

In respect of some residents however, the incentive or mandate accompanied with training might be insufficient to motivate sustained completion. For example, this might apply to people at the end of life, possibly because staff knew that residents' deterioration was irreversible (Table 1).

The motivation of frontline care staff to complete resident data in a systematic way may result in less burdensome assessment (for example by reducing duplication) releasing more time for frontline care staff to focus on resident care (Table 1: C-CMOs 2 and 4B).

\section{Frontline staff monitoring}

The process of MDS completion and personnel involved affect how residents' needs are captured and the impact this has on the working of the care home. To collect 
resident data in a systematic way, frontline staff must monitor their data collection (Fig. 4: C-CMO 5). This self-reflexive monitoring is supported by receiving feedback by health care professionals. At the resident level of care, under-reporting (or omission) of information was linked to carers relying on their own judgement and interpretation of the intensity of residents' experiences. This was evident when residents could not communicate their needs, for example in relation to pain and depression $[23,25,53]$. For an MDS to operate requires the regular involvement and engagement of health care professionals both within and from outside the care home (C-CMOs 6A) who are prioritising resident needs, direct care planning, discussing and reviewing progress with care staff (C-CMOs 4A, 4B, 5).

The MDS values standardising and creating a common language between different practitioners both visiting and caring for residents. There was an underlying tension about whose language and categorisation dominated. One stakeholder interview had described how the perceived medical orientation of the MDS affected the detail staff might record or how they would use it if their understanding of the resident did not fit with the categorisation. Key to effective monitoring is ensuring the MDS allows care home staff involved in direct care to share with others their observations and personal insights (e.g., change of shift reports, family conferences) [22, $54,55]$. Structured opportunities within the MDS to share this kind of information are an important context that respects and values the knowledge of all care staff and not just health care professionals. Studies of MDS implementation described that care staff and health care professionals needed to have ongoing conversations about case load organisation and the significance of what was being recorded to support ongoing engagement in using the MDS (Table 1).

Completing a typical MDS is time-consuming. Where IT literate staff are using digital assessments, this helps them to receive and respond to feedback from clinical staff, and thus to increase their self-monitoring and systematic completion of an MDS (Fig. 4: C-CMOs 1A, 5, $6 \mathrm{~A}, 6 \mathrm{~B}, 6 \mathrm{C})$. The availability of designated staff with protected time to complete an MDS is compromised by staff shortages and turnover $[56,57]$ (also see Table 1 ). One early study found that the resident's first assessment takes 60-90 minutes to complete [22]. Over two decades since that report, the time required is similar [23, 58-61]. This links to our first proposition and the need for training and resources to create a shared understanding and motivation to use the MDS as the basis for care.

\section{Embedded recording systems}

Digital assessment tools used in real time with residents by IT literate staff seemed important for MDS completion (C-CMOs 6A, 6B, 6C, 9, 1A). Care homes with decreased uptake of health information technologies were theorised to experience decreased benefits from an MDS. The stakeholder interviews and scoping review suggested this went beyond a recognition that being comfortable with information technology (IT) affects uptake and use of an MDS. A quality manager of a care organisation argued when interviewed that most care staff (80-90 percent) wanted to provide care rather than spend time on technologies. There was little theory around this in the identified sources or further purposive searches of the literature. The theory developed in this review points to the importance of care-home level actors, usually care home staff, implementing digital tools in ways that create dialogue with all staff throughout piloting and implementing these tools (C-CMOs 6B, 6C, 9). When all care home staff were actively engaged with electronic documentation systems, the detail of residents' care improved, for example, for residents experiencing incontinence $[62,63]$. Equally, adoption of IT and losing the opportunity to discuss and receive information face to face about residents' care affects whose information is included or excluded, and who can access (or not) information [55]. Balancing the preferences of staff and the use of IT at the point of care are important [63, 64].

Care home readiness to implement digital tools will also play a critical role (CCMO 96B), which will depend upon care home capacity (CCMO 6B) size and the culture of inclusion and of having a common goal (CCMO 9). When it was clear that integrating an IT based MDS into daily care routines supported care processes and benefitted residents, their families, and staff, there were improved resident outcomes in key areas; for example, activities of daily living and in residents physical activity [65]. How other sources of information and use of parallel systems of recording information (e.g. paper records) diminish will affect the embedding of an MDS and if it captures nursing home residents' experience of care [20, 55]. A Belgian study found it took a year to integrate the InterRAI Palliative Care instrument into the day-to-day practices of a nursing home [23].

\section{Discussion}

This review explains how the uptake and use of MDS may improve outcomes for staff and residents, and in what circumstances to provide an account of how an MDS can support residents' care in settings with no prior history of its use.

From the outset we knew that mandates and incentives were a key context and that bottom-up and top-down 
approaches were needed to converge for effective and successful implementation [66]. Reform is more likely when policies are viewed as clinically relevant, coherent and achievable, then the regulations become more sustainable over time $[67,68]$.

Triggering of responses that lead to changes in residents' assessment and care needed additional contexts; training for all members of staff on the significance of different assessment categories for direct care and ongoing involvement of clinicians in the assessment and review of resident data. These generate a sense of collective purpose, understanding and recognition of the value of using residents' data to inform care. There are implications for how this is funded and if all care homes and their staff have equal access to this level of support and training. A key finding that appeared to be specific to the long-term care workforce, was the importance of staff confidence when both entering and using data, and if entering and reviewing resident data were perceived as separate to, and a distraction from, care work.

These findings resonates with the four domains of the Normalisation Process Theory (NPT) (collective action, coherence, cognitive participation and reflexive monitoring) and how meaningful change occurred when the relevant actors were persuaded that the new system would be as good if not better, in a context where the imperative to make a change was externally imposed [69]. The review demonstrated the human processes and responses involved when seeking to "normalise" the MDS into the work of the care home. Key, was investing in training that went beyond equipping staff to be skilled in data capture to enable a shared understanding (coherence) about why they would want to use information from an MDS when discussing residents and making decisions.

Advocates of an MDS argue that standardised forms of assessment provide a foundation for ongoing care, contributing to understanding residents' needs and early identification of potential risks and problems. This is grounded in theories of multidisciplinary working and what supports continuity of care for older people with complex and varied needs based on evidence of what works [70-72]. Evidence of the impact of multidisciplinary team working in care homes demonstrate improvement in patient assessment and management practices, including responsive behaviours, falls, use of antipsychotics, depressive symptoms, appropriateness of medications, restraint use, nutrition, and pain [73-75]. For an MDS to be able to exploit these known benefits of multidisciplinary working, staff training should follow the same approach, with all types and grades of staff learning to use MDS together creating opportunities to contribute their different knowledge about the resident. Having a member of staff responsible for how an MDS was completed was important to ensure completion and protected staff time. However, without structured opportunities for everyone to contribute and residents' priorities to be included, it could disenfranchise the knowledge of those giving and receiving the care and introduce a false divide between those who controlled the information and those who did not. As a resource, how MDS adoption and use are introduced links to social identity theories and research on what fosters a shared approach and agreement around goals and values for providing care in long term settings [76]. In long term care, staffs' social identity and engagement, in this case with an MDS, needs to demonstrate how it meaningfully supports specific norms, and values about residents' care. Interventions to encourage the uptake of an MDS that support identity mobilisation and working practices that can reinforce group relations are the drivers for uptake and change. Without this, involvement in an MDS risks being a distraction and threat to the groups' values of how they define and value their care work. By valuing the contributions of staff and building on existing ways of working, the uptake and use of an MDS has the potential to enable all staff to learn with and from each other about what is important for residents and their care.

Despite the extensive use of MDS as a data source for commissioning and research, most papers that relied on these data were excluded, because of the absence of discussion in the papers about the quality of the data, how it was entered and learning about underrepresented groups and missing data.

The evidence in this review supported the importance of ongoing engagement of health professionals from outside of the care home, for example geriatricians [73, $77,78]$. The detail did not allow us to conclude if a mix or a particular professional group were needed to enable MDS uptake.

Care homes have characteristics that affect uptake and use of innovation [77-81]. At the resident level of care, we know care home staff have limited access to training, low pay, and a high turnover of employment [79, 82, 83]. There is little or no evidence of the efficacy of stand-alone care home staff training unless it is linked to mechanisms of 'reinforcing' (e.g., additional supervision or individual skills training), or 'enabling' (e.g., help to put learning into practice) [84]. Arguably, the daily use of the MDS, training and clinician engagement described in the few implementation studies retrieved could trigger these responses. There was, however, no agreement about how long was required to ensure engagement with an MDS and what ensured that staff involved in care habitually used the MDS. Nor was it discussed if the length of time between staff entering resident information and it influencing decisions about residents' care 
affected how staff subsequently used the information for discussions and feedback to staff on resident outcomes. Claims that staff could be supported to use the MDS in a few sessions do not fit with studies that have required cross care home engagement and staff participation to change practice [85-87]. Research testing theories of goal setting has demonstrated that communications in care homes can be improved by providing feedback, guided by goal-setting theory and that highly resource-intensive feedback interventions may be unnecessary [54]. To be effective however, this would need an individual's goals to include gaining skills in using an MDS and organisational support.

The challenges of IT implementation are well documented, but this review raised questions if characteristics of the care home workforce and the care home location in relation to other systems of care affected uptake and use of MDS. The relationship between nursing, senior and junior care staff within the care home, prior experience, level of anxiety about IT and type of responsibilities, are issues identified by other authors in other mixed care settings as affecting staff engagement [88]. Depending on who was confident enough to use MDS and the supporting technology, who had permission to use it and opportunity to inform the MDS either created a sense of shared endeavour or led to parallel systems of information exchange for the purposes of care. Linked to this was how resident data could be shared to inform care with outside organisations. COVID-19 in the UK has exposed the difficulties of linking data on residents to inform decision making and the need for digital integration is a recognised priority [8-10].

The proposed programme theory is constrained by the evidence that was available and the inferences that could be made from the data. Available evidence clustered around the training for staff, their actions and organisational support. There was less evidence on how MDS use facilitated communication within the care home and linked outcomes at the resident level of care.

\section{Strengths, limitations, and future research directions}

This review asks how MDS can be implemented in care home settings. The strength of realist approaches is the focus on how different contexts can generate different responses from participants and so outcomes. It is a strength of this review that it articulates the contexts and care home specific mechanisms that are likely to lead to uptake of MDS at the resident level of care.

Most of the evidence was North American, and the organisation and structure of the surrounding systems of health care were not explored in this review. A study in England argued that recognising the relationships care homes have with other providers, and the wraparound care received from external health care providers, directly affected residents' experience of care and access to medical support [89].

There is no evidence presented in this review about the wider narratives and discourses around nationally deployed social care information systems and/or minimum data sets. Greenhalgh and colleagues suggest that for the application of technologies, creating an effective inter-stakeholder dialogue and building learning communities are necessary in realising a focal community idea [90]. This could be relevant in addressing our finding about what needs to be in place for the introduction of electronic forms of minimum data sets in the care sector at a wider level. The learning from the pandemic has meant that there is a greater openness to discussing change and how to share information within and across health and social care.

\section{Conclusion}

Research has demonstrated the value of MDS to commissioners and service providers in the identification of care needs. This review focused on how its use on a day-to-day basis could influence care work and resident outcomes. A national/federal mandate is highly relevant for the success of an MDS, but not always meaningful or beneficial to the staff who provide care. If, however, it is implemented with training and clinician engagement, its use can be a key motivator for improving day to day resident care and outcomes, as well as regional and national understanding of the care home population.

This analysis enables us to articulate how data informed discussions about residents can be normalised by focusing on the working environment of the care home and the way in which an MDS is introduced, discussed and used over time. It directs attention to the important issue of how to tailor and implement an MDS likely to inform residents' everyday care, by identifying the causal mechanisms of, prioritising data capture, staff and clinician engagement, and staff confidence and the contexts that enable them. Achieving this requires resources: funding and time to support staff training and strategies that sustain engagement and motivation from staff and visiting practitioners. This will ensure that resident data in an MDS are valid and valued by care home staff as an aid for care rather than an administrative burden.

\section{Abbreviations}

CMO: Context Mechanism Outcome; InterRAI: LTCF: International Resident Assessment Instrument Long Term Care Facility; MDS: Minimum Data Set. 


\section{Supplementary Information}

The online version contains supplementary material available at https://doi. org/10.1186/s12877-021-02705-W.

Additional file 1. Interview schedules.

Additional file 2. Example of search terms used across databases to retrieve relevant literature.

Additional file 3. If-then statements.

Additional file 4. Sources of papers per countries.

Additional file 5. In-depth programme theory diagram to supplement Fig. 4.

\section{Acknowledgements}

We would like to thank the DACHA Patient and Public Involvement and Engagement panel for their comments on the review and discussion of the findings and implications for care home use and uptake by staff, residents and their representatives.

\section{Authors' contributions}

Concept and design of the review are embedded in the Developing research resources And minimum data set for Care Homes' Adoption and use (DACHA) study. SK conducted the initial database literature search and CG SB MKM, GA, wrote the first draft of the manuscript. Critical review, commentary on drafts and refinement of the manuscript were provided by $A K, K S, G P, J K B, A L G, B H$, AMT, LI, $\amalg$ and JM. MKM and CG wrote the final version of the manuscript. All authors have read and approved the manuscript

\section{Funding}

The DACHA study is funded by the National Institute for Health Research Health Service \& Delivery Research programme (NIHR HS\&DR project number NIHR127234) and supported by NIHR Applied Research Collaborations (East of England, East Midlands, Yorkshire and Humber, North East and Cumbria). Claire Goodman is an NIHR Senior Investigator. The views and opinions expressed therein are those of the authors and do not necessarily reflect those of the NIHR HS\&DR, ARC, NIHR, NHS or the Department of Health and Social Care.

\section{Availability of data and materials}

The data generated and analysed during the current review are not suitable for sharing beyond that contained within the report. Further information can be obtained from the corresponding author on reasonable request.

\section{Declarations}

\section{Ethics approval and consent to participate}

The University of Hertfordshire Ethics Committee approved this study (HSK SF/UH/04169). Written informed consent was obtained prior to stakeholder interviews.

\section{Consent for publication \\ Not applicable}

\section{Competing interests}

The authors declare they have no competing interests.

\section{Author details}

${ }^{1}$ Centre for Research in Public health and Community Care (CRIPACC), School of Health and Social Work, University of Hertfordshire, Hatfield, UK. ${ }^{2}$ National Institute for Health Research Applied Research Collaboration South West Peninsula (PenARC), University of Exeter Medical School, St Luke's Campus, Heavitree Road, Exeter, UK. ${ }^{3}$ Faculty of Medicine and Health Sciences, University of East Anglia, Norwich, UK. ${ }^{4}$ School of Healthcare, University of Leeds, Leeds, UK. ${ }^{5}$ NIHR Applied Research Collaboration, Yorkshire and Humber, Leeds, UK. ${ }^{6}$ Institute of Cardiovascular and Medical Sciences, University of Glasgow, Glasgow, UK. ${ }^{7}$ School of Medicine, University of Nottingham, Nottingham, UK. ${ }^{8} \mathrm{NIHR}$ Applied Research Collaboration, East Midlands (ARC-EM), Leicester, UK. ${ }^{9}$ Population Health Sciences Institute, Campus for Ageing and Vitality,
Newcastle University, Newcastle upon Tyne, UK. ${ }^{10}$ NIHR Applied Research Collaboration, North East and North Cumbria, Newcastle, UK. ${ }^{11}$ Centre for Health Services Studies, University of Kent, Canterbury, UK. ${ }^{12}$ NIHR Applied Research Collaboration, Surrey and Sussex, Kent, UK. ${ }^{13}$ Cambridge Public Health, University of Cambridge, Cambridge, UK. ${ }^{14}$ National Care Forum, Friars House, Manor House Drive, Coventry, UK. ${ }^{15}$ Care for Older People, School of Health Sciences, Division of Nursing, City, University of London, London, UK. ${ }^{16} \mathrm{NIHR}$ Applied Research Collaboration East of England, Cambridge, UK.

Received: 14 June 2021 Accepted: 7 December 2021

Published online: 07 January 2022

\section{References}

1. Later life in the United Kingdom 2019 [https://www.ageuk.org.uk/ globalassets/age-uk/documents/reports-and-publications/later_life_ uk_factsheet.pdf]

2. World Report on Ageing and Health [https://apps.who.int/iris/bitst ream/handle/10665/186463/9789240694811_eng.pdf?sequence=1]

3. The state of health care and adult social care in England 2016/17 [https:// www.cqc.org.uk/sites/default/files/20171011_stateofcare1617_summary. pdf ]

4. Kingston A, Commas-Herrera A, Jagger C. Forecasting the care needs of the older population in England over the next 20 years: estimates from the Population Ageing and Care Simulation (PACSim) modelling study. Lancet Public Health. 2018;3(9):e447-55.

5. Underwood M, Lamb SE, Eldridge S, Sheehan B, Slowther A, Spencer A, et al. Exercise for depression in care home residents: a randomised controlled trial with cost-effectiveness analysis (OPERA). Health Technol Assess (Winchester, England). 2013;17(18):1-281.

6. Facts for the media [https://www.ageuk.org.uk/globalassets/age-uk/ documents/reports-and-publications/later_life_uk_factsheet.pdf]

7. Musa M, Saga S, Blekken L, Harris R, Goodman C, Norton C. The prevalence, incidence, and correlates of faecal incontinence among older people residing in care homes: a systematic review. J Am Med Dir Assoc. 2019;20:956-62.

8. Burton JK, Goodman C, Guthrie B, Gordon AL, Hanratty B, Quinn TJ. Closing the UK care home data gap - methodological challenges and solutions. Int J Popul Data Sci. 2020;5(4).

9. Gordon AL, Goodman C, Achterberg W, Barker RO, Burns E, Hanratty B, et al. Commentary: COVID in care homes-challenges and dilemmas in healthcare delivery. Age Ageing. 2020;49(5):701-5.

10. Hanratty B, Burton JK, Goodman C, Gordon AL, Spilsbury K. Covid-19 and lack of linked datasets for care homes. BMJ. 2020;369:m2463.

11. Hundreds of UK care home deaths not added to official coronavirus toll [https://www.theguardian.com/world/2020/apr/09/covid-19-hundredsof-uk-care-home-deaths-not-added-to-official-toll]

12. Daly M. COVID-19 and care homes in England: What happened and why? Soc Policy Adm. 2020;54(7):985-98.

13. Smith CM, Williams $H$, Jhass A, Patel $S$, Crayton E, Lorencatto F, et al. Antibiotic prescribing in UK care homes 2016-2017: retrospective cohort study of linked data. BMC Health Serv Res. 2020;20(1):555.

14. Hirdes JP, Ljunggren G, Morris JN, Frijters DHM, Finne Soveri H, Gray $L$, et al. Reliability of the interRAl suite of assessment instruments: a 12 -country study of an integrated health information system. BMC Health Serv Res. 2008;8(1):277.

15. Saliba D, Buchanan JL. Making the investment count: revision of the Minimum Data Set for nursing homes, MDS 3.0. J Am Med Dir Assoc. 2012;13(7):602-10.

16. Hutchinson A, Milke D, Malsey S, Johnson C, Squires J, Teare G, et al. The resident assessment instrument-minimum data set 2.0 quality indicators: a systematic review. Health Serv Res. 2020;10:1-14.

17. Revised Long-Term Care Facility Resident Assessment Instrument User's Manual Version 2.0 [http://www.hpm.umn.edu/nhregsplus/Resources\% 20and\%20Publications/Federal_Resources/RAl/Complete\%20RAl.pdf]

18. Chen L, Liu L, Peng L, Lin M, Chen L, Lan C, et al. Identifying residents at greater risk for cognitive decline by Minimum Data Set in long-term care settings. J Clin Gerontol Geriatrics. 2014;5(4):122-6.

19. Dash D, Heckman GA, Boscart VM, Costa AP, Killingbeck J, d'Avernas JR. Using powerful data from the interRAI MDS to support care and a 
learning health system: A case study from long-term care. Healthcare Management Forum. 2018;31(4):153-9.

20. Doupe M, Pass J. J N, Garland A, Dik N, Zinnick S, Lix L: How well does the minimum data set measure healthcare use? a validation study. BMC Health Serv Res. 2018;18.

21. Gleeny C, Stolee P. Comparing the functional independence measure and the interRAl/MDS for use in the functional assessment of older adults: a review of the literature. BMC Getriatrics. 2009;9(52):1-12.

22. Hansebo G, Kihlgren M, Ljunggren $G$, Winblad B. Staff views on the Resident Assessment Instrument, RAI/MDS, in nursing homes, and the use of the Cognitive Performance Scale, CPS, in different levels of care in Stockholm. Sweden J Advan Nursing. 1998;28(3):642-53.

23. Hermans K, De Almeida MJ, Spruytte N, Cohen J, Van Audenhove C, Declercq A. Does using the interRAI Palliative Care instrument reduce the needs and symptoms of nursing home residents receiving palliative care? Palliative Supportive Care. 2018;16(1):32-40.

24. Wysocki A, Thomas K, Mor V. Functional Improvement Among ShortStay Nursing Home Residents in the MDS 3.0. J Am Med Dir Assoc. 2015;16(6):470-4.

25. Armstrong H, Daly T, Choiniere J. Policies and practices: The case of RAlMDS in Canadian long-term care homes. J Can Stud. 2016;50(2):348-67.

26. Hirdes JP, Poss JW, Caldarelli H, Fries BE, Morris JN, Teare GF, et al. An evaluation of data quality in Canada's Continuing Care Reporting System (CCRS): secondary analyses of Ontario data submitted between 1996 and 2011. BMC Med Inform Decision Making. 2013;13:27.

27. Onder G, Carpenter I, Finne-Soveri H, Frijters D, Henard J, Nikolaus T, et al. Assessment of nursing home residents in Europe: the Services and Health for Elderly in Long TERm care (SHELTER) study. BMC Health Serv Res. 2012;12(5):1-10.

28. Developing the use of MDS/RAI reports for UK care homes [https://core. ac.uk/download/pdf/64174.pdf]

29. Using interRAl assessment systems to measure and maintain quality of long-term care [https://www.interrai.org/assets/files/par-i-chapter-3-oldage.pdf]

30. Abayneh S, Lempp H, Manthorpe J, Hanlon C. Development of programme theory for integration of service user and caregiver involvement in mental health system strengthening: protocol for realist systematic review. Int J Ment Heal Syst. 2018;12:41-1.

31. Maidment ID, Lawson S, Wong G, Booth A, Watson A, McKeown J, Zaman $\mathrm{H}$, Mullan J, Bailey S: Medication management in older people: the MEMORABLE realist synthesis. 2020, 8:26.

32. Wong G, Westhorp G, Manzano A, Greenhalgh J, Jagosh J, Greenhalgh T. RAMESES II reporting standards for realist evaluations. BMC Med. 2016;14(1):96

33. Wong G, Westhorp G, Pawson R, Greenhalgh T. Realist Synthesis: RAMESES Training Mat. 2013.

34. Realist synthesis: an introduction [https://www.researchgate.net/profile/ Gill_Harvey/publication/228855827_Realist_Synthesis_An_Introduction/ links/Ofcfd507f0b7cbb2ce000000.pdf]

35. Pawson R. Tilley: An introduction to scientific realist evaluation. Thousand Oaks, CA: SAGE; 1997.

36. Rees CE, Lee SL, Huang E, Denniston C, Edouard V, Pope K, et al. Supervision training in healthcare: a realist synthesis. Adv Health Sci Educ. 2020;25(3):523-61.

37. Wong G, Greenhalgh T, Westhrop G, Pawson R. Development of methodological guidance, publication standards and training materials for realist and meta-narrative reviews: the RAMESES (Realist And Meta-narrative Evidence Syntheses - Evolving Standards) project. Health Serv Deliv Res. 2014;2.

38. Musa MK, Akdur G, Hanratty B, Kelly S, Gordon A, Peryer G, et al. Uptake and use of a minimum data set (MDS) for older people living and dying in care homes in England: a realist review protocol. BMJ Open 2020;10(11):e040397.

39. Jagosh J, Pluye P, Wong G, Cargo M, Salberg J, Bush P, et al. Critical reflections on realist review: insights from customizing the methodology to the needs of participatory research assessment. Res Synth Methods. 2014;5(2):131-41.

40. Astbury B, Leeuw FL. Unpacking Black Boxes: Mechanisms and Theory Building in Evaluation. Am J Eval. 2010;31(3):363-81.

41. Sims S, Leamy M, Davies N, Schnitzler K, Levenson R, Mayer F, et al. Realist synthesis of intentional rounding in hospital wards: exploring the evidence of what works, for whom, in what circumstances and why. BMJ Qual Saf. 2018:27(9):743.

42. De Weger E, Van Vooren NJE, Wong G, Dalkin S, Marchal B, Drewes HW, et al. What's in a Realist Configuration? Deciding Which Causal Configurations to Use, How, and Why. International Journal of Qualitative. Methods. 2020;19:1-8.

43. Devi R, Hinsliff-Smith K, Goodman C, Gordon AL. The Covid-19 pandemic in UK care homes - revealing the cracks in the system. J Nursing Home Res. 2020;6:58-60.

44. Rycroft-Malone J, Burton C, Hall B, McCormack B, Nutley S, Seddon D, et al. Improving skills and care standards in the support workforce for older people: a realist review. BMJ Open. 2014;4(5):e005356.

45. Greenhalgh J, Pawson R, Wright J, Black N, Valderas JM, Meads D, et al. Functionality and feedback: a protocol for a realist synthesis of the collation, interpretation and utilisation of PROMs data to improve patient care. BMJ Open. 2014;4(7):e005601.

46. Rahman M, Foster AD. Racial segregation and quality of care disparity in US nursing homes. J Health Econ. 2015:39:1-16.

47. Rivera-Hernandez M, Kumar A, Epstein-Lubow G, Thomas KS. Disparities in Nursing Home Use and Quality Among African American, Hispanic, and White Medicare Residents With Alzheimer's Disease and Related Dementias. J Aging Health. 2019;31 (7):1259-77.

48. Shippee TP, Ng W, Bowblis JR. Does Living in a Higher Proportion Minority Facility Improve Quality of Life for Racial/Ethnic Minority Residents in Nursing Homes? Innovation. Aging. 2020;4(3).

49. Sherman M. An exploration of the experiences of registered nurses working in aged residential care facilities regarding interRAl: A qualitative research design. Master of Health Sciences: University of Otago; 2020.

50. Rahman A, Applebaum A. The nursing home minimum data set assessment instrument: manifest functions and unintended consequences - past, present, and future. The Gerontologist. 2009:49(6):727-35.

51. Hoben M, Yoon MN, Lu L, Estabrooks CA. If we cannot measure it, we cannot improve it: Understanding measurement problems in routine oral/ dental assessments in Canadian nursing homes-Part I. Gerodontology. 2020;37(2):153-63.

52. Poss JW, Jutan NM, Hirdes JP, Fries BE, Morris JN, Teare GF, et al. A review of evidence on the reliability and validity of Minimum Data Set data. Healthcare Manag Forum. 2008;21(1):33-9.

53. Hyun J, Schere Y. Advantages and disadvantages of using MDS data in nursing home research. J Gerotol Nursing. 2009;35(1):7-17.

54. Hoben $M$, Ginsburg LR, Easterbrook A, Norton PG, Anderson RA, Andersen EA, et al. Comparing effects of two higher intensity feedback interventions with simple feedback on improving staff communication in nursing homes-the INFORM cluster-randomized controlled trial. Implement Sci. 2020;15(1):75.

55. Kontos PC, Miller K-L, Mitchell GJ. Neglecting the importance of the decision making and care regimes of personal support workers: a critique of standardization of care planning through the RAI/MDS. Gerontologist. 2010;50(3):352-62.

56. Ersek M, Polissar N, Neradilek MB. Development of a composite pain measure for persons with advanced dementia: exploratory analyses in self-reporting nursing home residents. J Pain Symptom Manag. 2011:41(3):566-79.

57. Fedecostante M, Dell'Aquila G, Eusebi P, Volpato S, Zuliani G, Abete P, et al. Predictors of Functional Changes in Italian Nursing Home Residents: The U.L.I.S.S.E. Study J Am Med Dir Assoc. 2016;17(4):306-11.

58. Devriendt E, Wellens N, Flamming J, Declerca A, Moons P, Boonen S, et al. The interRAI Acute Care instrument incorporated in an eHealth system for standardized and web-based geriatric assessment: strengths, weaknesses, opportunities and threats in the acute hospital setting. BMC Getriatrics. 2013;13(1).

59. Hermans K, Spruytte N, Cohen J, Van Audenhove C, Declercq A. Usefulness, feasibility and face validity of the interRAI Palliative Care instrument according to care professionals in nursing homes: A qualitative study. Int $J$ Nurs Stud. 2016;62:90-9.

60. Shin $\mathrm{H}$, Schere $Y$. Advantages and disadvantages of using MDS data in nursing home research. J Gerotol Nursing. 2009;35(1):7-17.

61. Vanneste D, Declercq A: The Development of BelRAl, a Web Application for Sharing Assessment Data on Frail Older People in Home Care, Nursing Homes, and Hospitals. In: Achieving Effective Integrated E-Care Beyond the 
Silos. Edited by Meyer I, Müller S, Kubitschke L. Hershey, PA: IGI Global; 2014.

62. Alexander GL, Madsen R. IT sophistication and quality measures in nursing homes. J Gerontol Nurs. 2009;35(7):22-7.

63. Alexander GL, Pasupathy KS, Steege LM, Strecker EB, Carley KM. Multidisciplinary communication networks for skin risk assessment in nursing homes with high IT sophistication. Int J Med Inform. 2014;83(8):581-91.

64. Huang Y-H, Garrett SK. Defining characteristics of communication quality in culture-changed long-term healthcare facilities. J Commun Healthcare. 2012;5(4):227-38.

65. Rantz MJ, Hicks L, Petroski GF, Madsen RW, Alexander G, Galambos C, et al. Cost, staffing and quality impact of bedside electronic medical record (EMR) in nursing homes. J Am Med Dir Assoc. 2010;11 (7):485-93.

66. From Anecdotes to Evidence: Complex Continuing Care at the Dawn of the Information Age in Ontario [http://www.milbank.org/reports/inter RAl/030222interRAl.html]

67. The Challenge of Dependency in a Changing Society: New Tools for a New Era at the Health Care-Social Care Interface in Spain [https://www. milbank.org/wp-content/files/documents/interRAl/030222interRAl.html]

68. Toward Informed and Evidence-based Elderly Care: The RAI Experience in Iceland [https://www.milbank.org/wp-content/files/documents/interRAl/ 030222interRAl.html]

69. Pope C, Halford S, Turnbull J, Prichard J, Calestani M, May C. Using computer decision support systems in NHS emergency and urgent care: ethnographic study using normalisation process theory. BMC Health Serv Res. 2013;13(1):111.

70. Role of comprehensive geriatric assessment in healthcare of older people in UK care homes: realist review [https://bmjopen.bmj.com/content/ bmjopen/9/4/e026921.full.pdf]

71. Freeman S, Hirdes JP, Stolee P, Garcia J, Smith TF, Steel K, et al. Care planning needs of palliative home care clients: Development of the interRAl palliative care assessment clinical assessment protocols (CAPs). BMC Palliative Care. 2014;13(1):58

72. Sales A, O'Rourke HM, Draper K, Teare GF, Maxwell C. Prioritizing information for quality improvement using resident assessment instrument data: experiences in one canadian province. Healthc Policy. 2011;6(3):55-69.

73. Barker RO, Craig D, Spiers G, Kunonga P, Hanratty B. Who Should Deliver Primary Care in Long-term Care Facilities to Optimize Resident Outcomes? A Systematic Review. J Am Med Dir Assoc. 2018;19(12):1069-79.

74. Nazir A, Unroe K, Tegeler M, Khan B, Azar J, Boustani M. Systematic review of interdisciplinary interventions in nursing homes. J Am Med Dir Assoc. 2013;14(7):471-8

75. Pillay B, Wootten AC, Crowe H, Corcoran N, Tran B, Bowden P, et al. The impact of multidisciplinary team meetings on patient assessment, management and outcomes in oncology settings: A systematic review of the literature. Cancer Treat Rev. 2016;42:56-72.

76. Amador S, Goodman C, Mathie E, Nicholson C. Evaluation of an Organisational Intervention to Promote Integrated Working between Health Services and Care Homes in the Delivery of End-of-Life Care for People with Dementia: Understanding the Change Process Using a Social Identity Approach. Int J Integr Care. 2016;16(2):14

77. Marshall EG, Clarke BS, Varatharasan N, Andrew MK. A Long-Term CareComprehensive Geriatric Assessment (LTC-CGA) Tool: Improving Care for Frail Older Adults? Can Geriatr J. 2015;18(1):2-10.

78. Panza F, Solfrizzi V, Lozupone M, Barulli MR, D'Urso F, Stallone R, et al. An Old Challenge with New Promises: A Systematic Review on Comprehensive Geriatric Assessment in Long-Term Care Facilities. Rejuvenation Res. 2018;21(1):3-14.

79. Bunn F, Goodman C, Corazzini K, Sharpe R, Handley M, Lynch J, et al. Setting Priorities to Inform Assessment of Care Homes' Readiness to Participate in Healthcare Innovation: A Systematic Mapping Review and Consensus Process. Int J Environ Res Public Health. 2020;17(3):987.

80. Estabrooks CA, Squires JE, Cummings GG, Birdsell JM, Norton PG. Development and assessment of the Alberta Context Tool. BMC Health Serv Res. 2009;9(1):234.

81. What we know about designing an effective improvement intervention (but too often fail to put into practice) [https://qualitysafety.bmj.com/ content/qhc/26/7/578.full.pdf]

82. Pitfield C, Shahriyarmolki K, Livingston G. A systematic review of stress in staff caring for people with dementia living in 24-hour care settings. Int Psychogeriatr. 2011;23(1):4-9.
83. Testad I, Mikkelsen A, Ballard C, Aarsland D. Health and well-being in care staff and their relations to organizational and psychosocial factors, care staff and resident factors in nursing homes. Int $\mathrm{J}$ Geriatric Psych. 2010;25(8):789-97.

84. Rapaport P, Livingston G, Murray J, Mulla A, Cooper C. Systematic review of the effective components of psychosocial interventions delivered by care home staff to people with dementia. BMJ Open. 2017:7(2):e014177.

85. Chenoweth L, King MT, Jeon YH, Brodaty H, Stein-Parbury J, Norman R, et al. Caring for Aged Dementia Care Resident Study (CADRES) of personcentred care, dementia-care mapping, and usual care in dementia: a cluster-randomised trial. Lancet Neurol. 2009;8(4):317-25.

86. Fossey J, Garrod L, Tolbol Froiland C, Ballard C, Lawrence V, Testad I. What influences the sustainability of an effective psychosocial intervention for people with dementia living in care homes? A 9 to 12-month followup of the perceptions of staff in care homes involved in the WHELD randomised controlled trail. Int J Geriatr Psychiatry. 2019;34(5):674-82.

87. Surr CA, Parveen S, Smith SJ, Drury M, Sass C, Burden S, et al. The barriers and facilitators to implementing dementia education and training in health and social care services: a mixed-methods study. BMC Health Serv Res. 2020;20(1):512.

88. Kuek A, Hakkennes S. Healthcare staff digital literacy levels and their attitudes towards information systems. Health Informatics $J$. 2020;26(1):592-612.

89. Goodman C, Davies S, Gordon A, Dening T, Gage H, Meyer J, et al. Optimal NHS service delivery to care homes: a realist evaluation of the features and mechanisms that support effective working for the continuing care of older people in residential settings. Health Services and Delivery Research. 2017;5(29).

90. Greenhalgh T, Procter R, Wherton J, Sugarhood P, Shaw S. The organising vision for telehealth and telecare: discourse analysis. BMJ Open. 2012;2(4):e001574.

\section{Publisher's Note}

Springer Nature remains neutral with regard to jurisdictional claims in published maps and institutional affiliations.

Ready to submit your research? Choose BMC and benefit from:

- fast, convenient online submission

- thorough peer review by experienced researchers in your field

- rapid publication on acceptance

- support for research data, including large and complex data types

- gold Open Access which fosters wider collaboration and increased citations

- maximum visibility for your research: over $100 \mathrm{M}$ website views per year

At BMC, research is always in progress.

Learn more biomedcentral.com/submissions 